\title{
Specificity of a cutaneous receptor
}

RONALD T. VERRILLO

LABORATORY OF SENSORY COMMUNICATION, SYRACUSE UNIVERSITY, SYRACUSE. NFW YORK

Ihi, luli vibrotactile thresholds are decrmined on the hummen Ipuqui and compared to corresponding dolo oblained

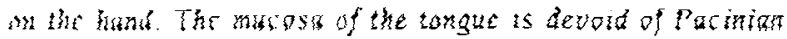
anpusics bue the hand is richly endowed with these nerve andings Summation of energy is charactcristic of responses rccordcd from tissue containing Pacinian corpuscles and abscme where the corpuscles do not exist. The argument is developed that the Pacinian corpuscle is specifically sensitive to mechanical vibration.

The doctrine of specific nerve energies and its counterpart of modality-specific peripheral terminations has been seriously challenged over the past decade (Sinclair, 1955). A number of contemporary reseaxchess sow favor pattern theories in which seural spectificity is replaced by the complex spatiat and temporal patterning of impulses (Melzack \& Wall, 1962). The present, study presents evidence that associated with cutaneous tissue are neural elements that are specifically sensitive to mechanical vibration.

The purpose of this experiment is to demonstrate that summation of energy over space and time is characteristic of responses from tissue containing Pacinian corpuscles and that these characteristics are lacking where the tissue is devoid of these encapsulations.

Two distinct forms of the threshold response are elicited from the mechanical stimulation of busman skin by sinusoidal signals, depending tpon the size of the contactor (Verrillo, 1963; in press). Large contactors yield U-shaped curves having a maximum of sensitivity at shout $250 \mathrm{cps}$ with a slope of $-12 \mathrm{dg}$ per doubling of frequency up to $250 \mathrm{cps}$ and about $+9 \mathrm{~dB}$ at higher frequencies. For very small contactors the curves are flat from 25 to $640 \mathrm{cps}$.

Spatial summation is evident at frequencies above $40 \mathrm{cps}$. Threshold values drop at the rate of $3 \mathrm{~dB}$ per doubling of contactor area indicating a complete integration of the energy increments delivered to the skin. At 25 and 40 cps the threshold curves are flat. These relationships are found in both hairy and glabrous skin. They led to the hypotiesha that cutaneous tissue is iost to at least two functionally distinct eypes of mechanoreceptors: one that summates energy over time and space and another that is unresponsive to changes in these parameters.

It is reasonable to suspect that the Pacinian corpuscle is the transducer responsible for the energy summation. The propagated impulses of Pacinian corpuscles are known to follow the frequency of sinusoidal mechanical stimulation in a 1:1 ratio within certain frequency limits (Scot1, 1951; Loewenstein, 1958; Hunt. 1961). It is also known that the threshold of excitation of these units varies as a function of stimulus frequency (S10, 1961). Although its resposse to mechanical stimulation may be affected by temperature variations, thermalchanges will not inifiate a spike potential (Loewenstein. 1961). Finally, a remarkable similarity exists between the threshold curves obtained by Sato (1961) from in situ preparations of Pacinian corpuscles in cat mesentery and curves obtained by psychophysical methods on humans (Verrillo, 1966).

Vibrotactile thresholds determined on a location known to be free of Pacinian corpuscles would help to clarify their role in mechanoreception. I chose the tongue as the sjte of stimulation for this study since it is generally agreed that the mucosa of the tongue contains no Pacinian corpuscles (Winkelmans, 1960; Gajrss, isaㅎ.

\section{Apparatus and Melhod}

The output of a sine-wave generator was modulated by an electronic switch to produce a signal that was on for $1 \mathrm{sec}$. and off for $1 \mathrm{sec}$. The signal was prearmplified and passed through a Békésy recording attenuator. After a final stage of amplification the signal activated the vibrator which was located within a sound-proof booth where the $S$ sat. The mechanical disturbance was limited to the immediate vicinity of the contactor by a rigid disk surrounding the contactor. The gap between the contactor and the disk was $2.0 \mathrm{~mm}$. It has been shown that such a device effectively prevents the spread of surface waves over the skin (Eijkman \& Vendrick, 1960). Greater detail of the apparatus can be obtained from previous publications (Verrillo, 1963; in press).

The stimulus was applied to the dorsal surface of the tongue just posterior to the tip. Comparison data for the hand were obtained on the palmar surface over the first metacarpus of the right hand. The Ss wore earphones with narrow band noise centered at $250 \mathrm{cps}$ to mask the vibrator signal which becomes audible at $250 \mathrm{cp} 5$. Each data point represents the median threshold of three Ss tested three limes each.

\section{Results}

Threshald responses obtained on the tongue and hand (Verrillo, 1963) are compared in Fig. 1. A contactor size of $1.3 \mathrm{~cm}^{2}$ was used in both experiments, It is obvious that for glabrous tissue, which has an abundance of Pacinian corpuscles, there is a dependence upon frequency over the range from 40 to $640 \mathrm{cps}$. Measurements on hairy skin results in a curve of similar shape (Verrillo, in press)。On the tongue, however, the curve is essentially flat; there is no summation of energy. The flat curve shown for the tongue (Fig. 1) does not 


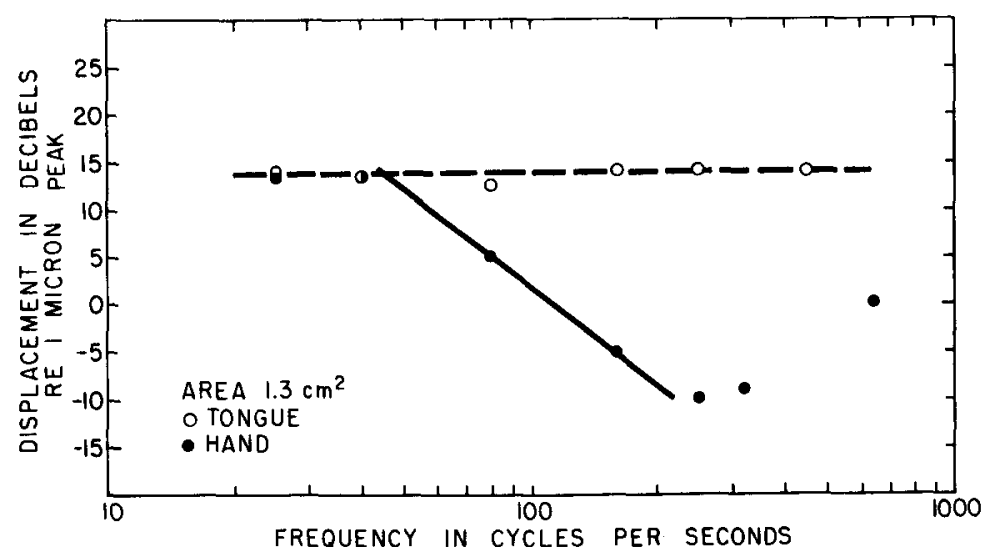

Fig. 1. Comparison of ahsolute vibrotactile thresholds for the hand and the tongue plotted as a function of stimulus frequency. agree with the U-shaped function obtained on the tongue by Sherrick (1953). There is an essential difference, however, in the conditions of the two experiments. Sherrick did not use a fixed disk around the contactor to prevent the spread of vibration to other parts of the tongue. The present experiments used the fixed disk. The main arteries and nerves serving the anterior portion of the tongue lie in the ventral quarter of the tongue and Pacinian corpuscles are known to occur in close proximity to all major nerves and blood vessels (Winkelmann, 1960). Thus, it is possible that the frequency dependence observed by Sherrick was determined by corpuscles located in tissues close to the underside of the tongue. This interpretation is consistent with the findings of Spassova (1965) who observed many GolgiMazzoni corpuscles deep in the musculature and along nerve bundles in the tongue of the cat. Only mucocutaneous end organs occurred in the dorsal aspect. Dogiel, Ruffini and Winkelmann regard the GolgiMazzoni body as Pacinian corpuscles modified by the mechanical characteristics of surrounding tissues (Winkelmann, 1960).

Thresholds were again determined on the dorsum of the tongue $\left(.08 \mathrm{~cm}^{2}\right.$ and $3.2 \mathrm{~cm}^{2}$ contactors) but without the limiting disk. A U-shaped curve was obtained, verifying Sherrick's results. If the vibratory disturbance is in fact spreading to the undersurface of the tongue, a similar curve should be obtained if measurements are made on the ventral surface. The tests were repeated on the undersurface of the tongue $\left(3.2 \mathrm{~cm}^{2}\right.$ contactor) with the limiting disk in place to prevent the spread of vibrations. The resulting curve (Fig. 2) closely resembles the function obtained on the dorsum under conditions that allow the disturbance to propagate freely from the intended site of stimulation. When the vibrations are confined to a location lacking Pacinian corpuscles (dorsum of tongue), the threshold is independent of the stimulus frequency. One would predict that Pacinian corpuscles are located in the ventral tissues of the human tongue.

Various contactor sizes were tested at a frequency of $160 \mathrm{cps}$. The curve for the hand (Fig. 3) has a slope of $-3 \mathrm{~dB}$ per doubling of the area, indicating that all of the stimulus energy is summated. The slope obtained on hairy skin is the same (Verrillo, in press). The corresponding data points measured on the tongue yield a flat function. There is no spatial summation on the dorsum of the tongue where Pacinian corpuscles do not exist.

In the second experiment the absolute threshold was measured as a function of the extent to which the contactor protruded into the tissue of the tongue. Earlier studies show that the threshold of vibration on the hand decreases as the resting position of the contactor is

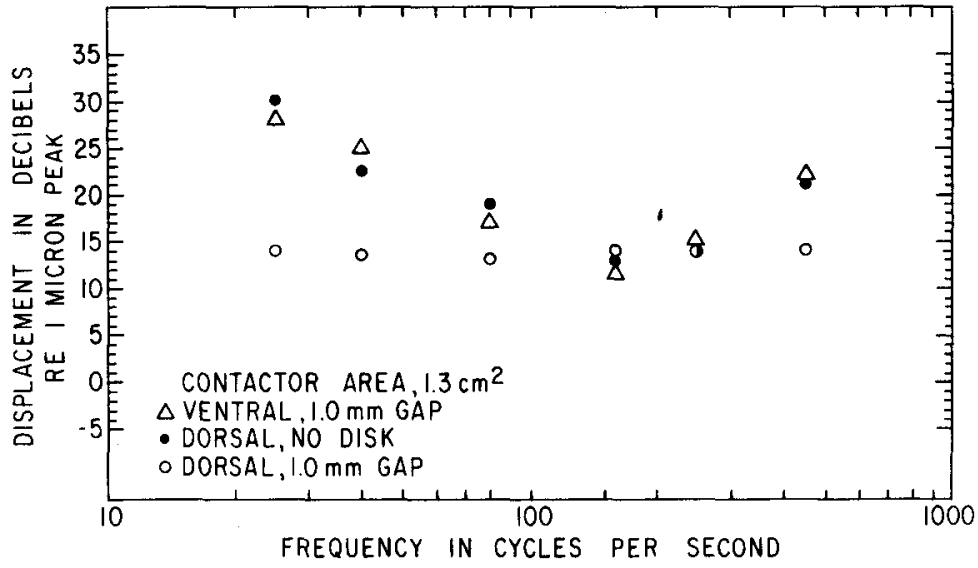

Fig. 2. Comparison of thresholds measured on the dorsal and ventral surfaces of the tongue 


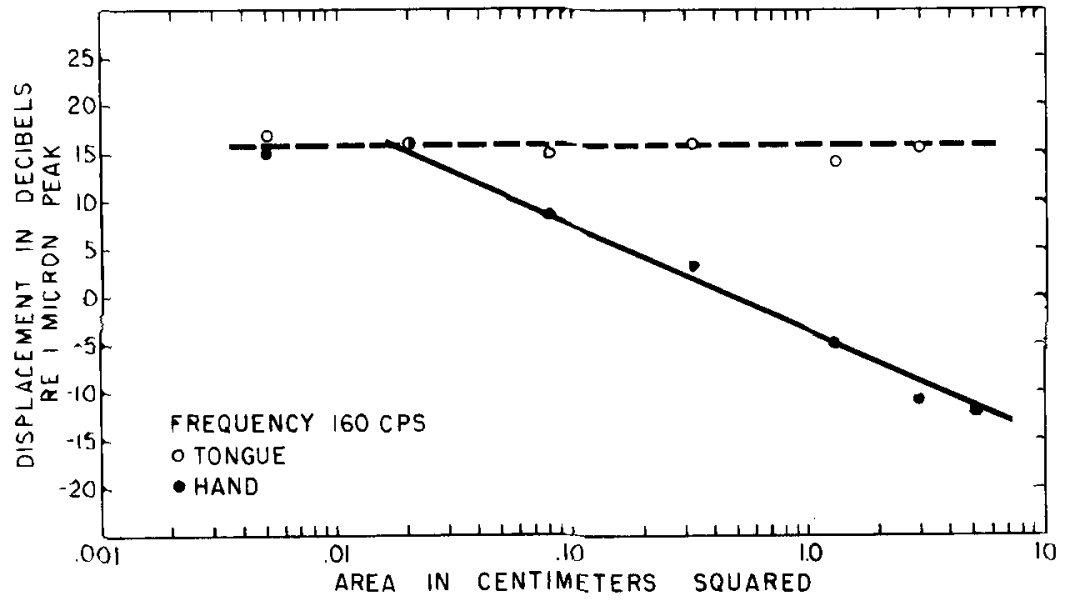

Fig. 3. Comparison of absolute vibrotactile thresholds for the hand and the tongue plotted as a function of contactor area. pressed deeper into the skin (Babkin et al, 1961; Verrillo, 1962). This effect has been observed on both hairy and glabrous skin. Moreover, the effect is greater at irequencjes above $40 \mathrm{cps}$. If the deep lying Pacinian corpuscle is a transducer of sinuscidal viorations, it is reasonable to expect that threshold would decrease as the stimulator is pressed closer to the sensitive unit.

Assuming that the Pacinian corpuscle is the frequency transducer, several predictions can be made. At a frequency of $160 \mathrm{cps}$ the corpuscle is capable of following a mechanical stimulus in a ratio of $1: 1$. Therefore, the threshold measured on the hand should decrease as a function of protrusion of the contactor. The corpuscle will not fire below $40 \mathrm{cps}$ (Hunt, 1961; Sato, 1961) since it responds to the slope of the stimulus velocity (Loewerstein \& Mendelson, 1964) and at irequencies below $40 \mathrm{cps}$ the critical slope is not reached. Therefore, the threshold should not change as a function of contactor height when measured at $25 \mathrm{cps}$ on the hand. On the tongue the threshold curve should be flat for both 25 and $160 \mathrm{cps}$ since the Pacinian corpuscle is not involved.

The results shown in Figs. 4 and 5 are entirely con-

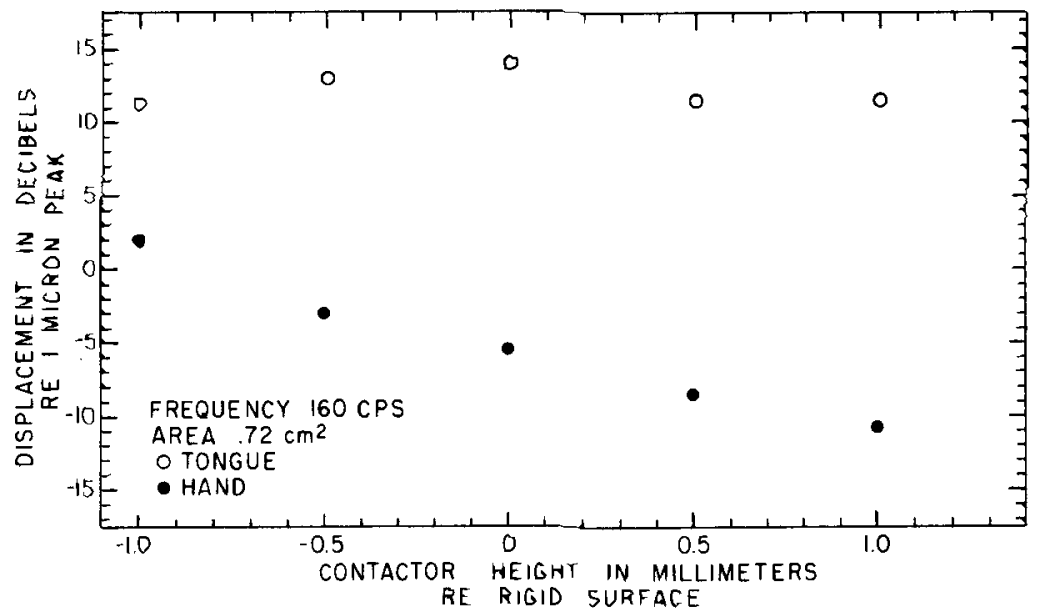

istent with these predictions. As the contactor height goes from negative to positive values, the contactor is pressed further into the skin. At zero height the contactor is fush with the table surface upon which the S's hand or tongue rests.

At 160 cps the pacinian corpuscle is responsive to mechanical stimulation and those tissues which contain underlying corpuscles (hand) show a decrease in threshold as the contactor presses deeper into the skin. The dorsum of the tongue, which has no corpuscles, yields a flat curve. At $25 \mathrm{cps}$ the curves are flat for both hand and tongue; in the hand the corpuscles cannot respond and in the tongue they are absent.

\section{Discussion and Conclusion}

Absolute thresholds for vibration were determined on the human tongue as a furction of stimulus 1 requency, contactor size and depth of protrusion of the contactor into the tissues. Previous investigations on glabrous and hairy skin show that thresholds obtained with larger contactors (.05 to $5.1 \mathrm{~cm}^{2}$ ) yield a consistent pattern when plotted as a function of frequency. The pattern cannot be obtained when testing is done with very small contactors $\left(.005\right.$ and $\left..02 \mathrm{~cm}^{2}\right)$. The two modes of response
Fig. 4. Absolute vibrotactile thresbolds for the hand and the tongue plotted as a function of contactor height. Thresholds were determined at a frequency of 150 cDS. 


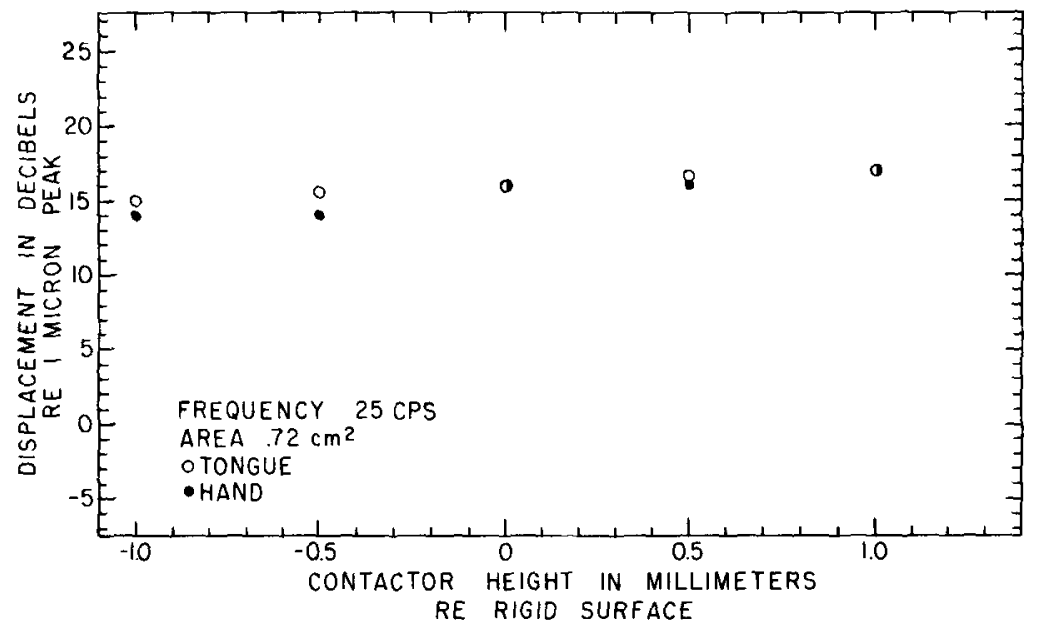

Fig. 5. Absolute vibrotactile thresholds for the hand and the tongue plotted as a function of contactor height. Thresholds were determined at a frequency of $25 \mathrm{cps}$. suggest that two types of mechanoreceptors may innervate cutaneous tissue. When plotted as a function of contactor size, thresholds on hairy and glabrous skin show spatial summation. Finally, as the resting level of the contactor is pressed more deeply into the skin, there is a corresponding decrease in vibrotactile thresholds.

The neural elements of glabrous skin consists of a dermal nerve net with fine, free endings, Meissner corpuscles, Merkel disks, and deep lying Pacinian corpuscles. ${ }^{2}$ Of these, the Pacinian corpuscle is the only one known to be capable of following the frequency of mechanical sinusoidal oscillations. The response pattern of individually tested units is remarkably similar to that of psychophysically measured thresholds obtained on glabrous skin.

Hairy skin contains these same neural elements with the exception of Meissner corpuscles. Since the frequency function for psychophysical thresholds on hairy skin closely follows that of glabrous skin, the Meissner ending can be eliminated as a possible frequency dependent ending. The hair-follicle nerve network can be ruled out as it is not found in glabrous skin. The Merkel disks are open to question: Winkelmann (1960) limits them to glabrous skin; Cauna (1962) finds them in glabrous and hairy skin; and Weddell et al (1954) regard them as artifacts. This leaves the dermal nerve net and the Pacinian corpuscles for consideration.

The dorsal tissue of the tongue are innervated by a dermal nerve net, mucocutaneous nerve endings and those nerves related to taste. The latter two types can be disregarded since they are not found in śkin where vibration sensitivity has been shown to exist. The important feature of the tongue is that it has the dermal nerve network with its free nerve endings and does not contain Pacinian corpuscles in the mucosa.

In the present investigations absolute thresholds obtained on the tongue when plotted as a function of stimulus frequency, contactor area and depth of protrusion into the tissue all yielded flat curves. The dermal nerve network can thus be dropped from con- sideration. This leaves only the Pacinian corpuscle.

Summation of energy over time and space and increasing sensitivity with contactor protrusion (the Pacinian corpuscle lies deep in the tissue) are characteristic of measurements made on tissues which contain Pacinian corpuscles. In the absence of Pacinian corpuscles, none of these characteristics can be produced.

On the basis of successive elimination of other cutaneous receptors and the direct evidence of physiological and psychological investigations it is concluded that the Pacinian corpuscle is a peripheral neural termination which functions specifically as a receptor for periodic mechanical stimuli.

\section{References}

Babkin, V. P., Rozen, O. M., Tumarkina, L. N., \& Chemyak, R. I. Investigation of vibration sensitivity and factors affecting it. Biophysics (trans Eiofizika), 1961, 6, 39-43.

Cauna, N. Functional significance of the submicroscopical, nistochemical and microscopical organization of the cutaneous receptor organs. Verhandl. Anat. Ges., 1962, 11, 181-197.

Eijkman, E., \& Vendrick, A. J. H. Dynamies of the vibration sense at low frequency. J. Acoust. Soc. Amer., 1960, 32, 1134-1139.

Gairns, F. W. Sensory nerve endings other than taste buds in the human tongue. J. Physiol, 1953, 121, 33p-34p.

Hunt, C. C. On the nature of vibration receptors in the hind limb of the cat. J. Physiol., 1961, 155, 175-186.

Loewenstein, $\boldsymbol{W}$. $\mathbf{R}$. Generator processes of repetitive activity in a Pacinian corpuscle, J. gen. Physiol., 1958, 41. 825-845.

Loewenstein. $\boldsymbol{W}$. $\mathbf{R}$. On the 'specificity' of a sensory receptor. J. Niurophysiol., 1961, 24, 150-158.

Loewenstein, $\boldsymbol{W}$. R., \& Mendelson, M. Components of receptor adaptation in a Pacinian corpuscle. J. Physiol., 1965, 17. 377-397.

Melzack, R., \& Wall, P. D. On the nature of cutaneous sellsory mechanisms. Brain, 1962, 85, 331-356.

Sato, M. Response of Pacinian corpuscles to sinusoidal vibration. J. Physiol., 1961, 159, 391-409.

Scott, D. Response of Pacinian corpuscles to oscillatory stimulation. Fed. Proc., 1951, 10, 123.

Sherrick, C. E., Jr. Variables affecting sensitivity of the Juman skin to mechanical vibration. J. c.tp. Puchol. 1953. 45. 273 282.

Spassova. I. On the structure of encapsulated nterve endings in the tongue of the cat and its functional significanct. $Z$. Mikr. anat. Forsch., 1965, 72, 366-382.

Sinclair, D. C. Cutaneous sensation and the doctrine of specific 
energy. Brain, 1955, 78, 584-614.

Verrillo, $\mathbf{R}$. $\mathbf{T}$. Investigation of some paranieters of the cutaneous threshold for vibration. J. Acoust. Soc. Amer., 1962, 34, 17681773.

Verrillo, R. T. Effect of contactor area on the vibrotactile threshold. J. Acoust. Soc. Amer., 1963, 35, 1962-1966.

Verrillo, R. T. Vibrotactile sensitivity and the frequency response of the Pacinian corpuscle. Psychon. Sci., 1966, 4, 135-136.

Verrillo, R. T. Vibrotactile thresholds for hairy skin. $J$. exp. Psychol., in press.

Weddell, G., Pallie, W., \& Palmer, Elizabeth. The morphology of peripheral nerve terminations in the skin. Quart. J. micr. Sci.,
$1954,95,483-501$

Winkelmann, R. K. Nerve endings in normal and pathologic skin. New York: Charles C. Thomas, 1960.

\section{Notss}

1. This work was supported by a contract between the National Institutes of Health, U. S. Department of Health Education and Welfare and Syracuse University.

2. The general schema of cutaneous neural innervation adhered to in this paper is based on the works of Winkelmann (1960) and Cauna (1962).

(Received in the Editorial Office March 15, 1966.) 